\title{
Time Varying Optimal Control with Packet Losses.
}

\author{
Bruno Sinopoli, Luca Schenato, Massimo Franceschetti, \\ Kameshwar Poolla, Shankar S. Sastry \\ Department of Electrical Engineering and Computer Sciences \\ University of California at Berkeley \\ \{sinopoli,lusche,massimof,sastry\}@eecs.berkeley.edu \\ poolla@me.berkeley.edu
}

\begin{abstract}
The problem of using wireless sensor networks technology for estimation and control of dynamical systems has recently received widespread attention within the scientific community. Classical control theory is in general insufficient to model distributed control problems where issues of communication delay, jitter, and time synchronization between components cannot be ignored.

The purpose of this paper is to extend our work on discrete time Kalman filtering with intermittent observations [1] that was motivated by data losses in a communication channel. Accordingly, we consider the Linear Gaussian Quadratic (LQG) optimal control problem in the discrete time setting, showing that the separation principle holds in the presence of data losses. Then, using our previous results, we show the existence of a critical arrival probability below which the resulting optimal controller fails to stabilize the system. This is done by providing analytic upper and lower bounds on the cost functional, and stochastically characterizing their convergence properties as $k \rightarrow \infty$.
\end{abstract}

\section{INTRODUCTION}

Advances in VLSI and MEMS technology have boosted the development of micro sensor integrated systems. Such systems combine computing, storage, radio technology, and energy source on a single chip [2] [3]. When distributed over a wide area, networks of sensors can perform a variety of tasks that range from environmental monitoring and military surveillance, to navigation and control of a moving vehicle [4] [5] [6]. A common feature of these systems is the presence of significant communication delays and data loss across the network. From the point of view of control theory, significant delay is equivalent to loss, as data needs to arrive to its destination in time to be used for control. In short, communication and control become tightly coupled such that the two issues cannot be addressed independently.

Consider, for example, the problem of navigating a vehicle based on the estimate from a sensor web of its current position and velocity. The measurements underlying this estimate can be lost or delayed due to the unreliability of the wireless links. What is the amount of data loss that the control loop can tolerate in order to reliably perform the navigation task? Can communication protocols be designed to satisfy this constraint? Practical advances in the design of these systems are described in [7]. The goal of this paper is to examine some control-theoretic implications of using sensor networks for control. These require a generalization of classical control techniques that explicitly take into account the stochastic nature of the communication channel.

In our setting, the sensor network provides observed data that are used to estimate the state of a controlled system, and this estimate is then used for control. However, due to unreliability of the network links, data communicated from the sensors to the estimator can be lost and we want to characterize the impact of such event on the control performance. Accordingly, we generalize the Linear Quadratic Gaussian (LQG) optimal control problem modeling the arrival of an observation as a random process whose parameters are related to the characteristics of the communication channel, as shown in Figure 1. The separation principle states that observer and plant of a linear system can be designed independently. We show that this principle continues to hold in the case of data loss between the sensor and the estimator. This allows to extend our results in [1], [8] showing the existence of a critical loss probability below which a transition occurs and the resulting optimal controller fails to stabilize the system. We show the existence of such transition by finding deterministic upper and lower bounds for the expected optimal cost and their convergence conditions.

In some related work [9] Nilsson presents the LQG optimal regulator with bounded delays between sensors and controller, and between the controller and the actuator, but he does not address the packet-loss case. This is considered by Hadijcostis and Touri [10]. Their analysis is restricted to the static scalar case. Other approaches include using the last received sample for control, or designing a dropout compensator [11], [12]. We consider the alternative approach where the external compensator feeding the controller is the optimal time varying Kalman gain. Moreover, we analyze the proposed solution in state space domain rather than in frequency domain as it was presented in [12], and we consider the more general Multiple Input Multiple Output (MIMO) case.

The LQG optimal control problem with missing observations can also be modelled using the well known Jump Linear System (JLS) theory [13], where the observer switches between open loop and closed loop configuration, depending on whether the packet containing the observation is lost, or arrives at the estimator in time. However, convergence results in this case can be obtained only when 


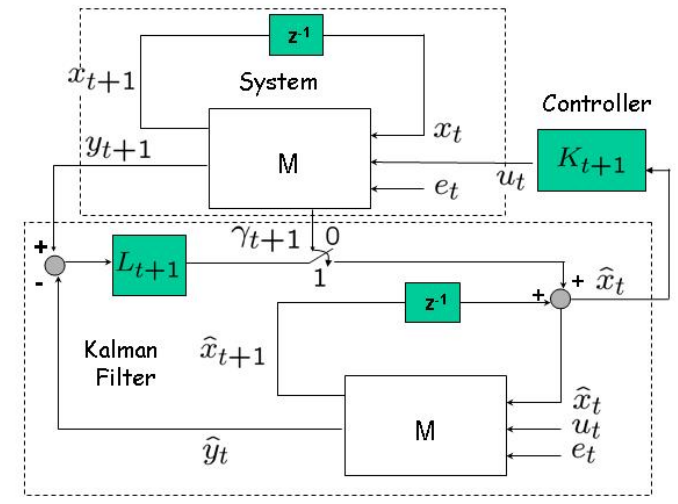

Fig. 1. Overview of the system. We study the statistical convergence of the expected state covariance of the discrete time LQG, where the observation, travelling over an unreliable communication channel, can be lost at each time step with probability $1-\bar{\gamma}$.

each jump sub-system is stabilizable and detectable. The detectability assumption fails in our case, producing a nonstationary state random process.

Finally, we mention that philosophically our result can be seen as another manifestation of the well known uncertainty threshold principle [14], [15]. This principle states that optimum long-range control of a dynamical system with uncertainty parameters is possible if and only if the uncertainty does not exceed a given threshold. The uncertainty is modelled as white noise scalar sequences acting on the system and control matrices. In our case the uncertainty is due to the random arrival of the observation, with the randomness arising from losses in the network.

The paper is organized as follows. In section II we formulate the LQG optimal control problem with intermittent observations. In section III we prove some lemmas we need to prove the main theorems. In Section IV we compute the optimal controller, showing that it is linear. We also provide upper and lower bounds on the cost functional of the LQG problem, and find the conditions on the observation arrival probability $\bar{\gamma}$ for which the upper bound converges to a fixed point, and for which the lower bound diverges. Finally, in section $\mathrm{V}$, we state our conclusions and give directions for future work.

\section{PROBlem FORMULATION}

Consider the following linear stochastic system with intermittent observations:

$$
\begin{aligned}
x_{k+1} & =A x_{k}+B u_{k}+w_{k} \\
y_{k} & =C x_{k}+v_{k},
\end{aligned}
$$

where $x_{k} \in \mathbb{R}^{n}$ is the state vector, $y_{k} \in \mathbb{R}^{m}$ is the output vector, $u_{k} \in \mathbb{R}^{q}$ is the input vector, $x_{0} \in \mathbb{R}^{n}, w_{k} \in \mathbb{R}^{n}$ and $v_{k} \in \mathbb{R}^{m}$ are Gaussian, uncorrelated, white, with zero mean and covariance $\left(P_{0}, Q, R_{k}\right)$ respectively, $R_{k}=\gamma_{k} R+(1-$ $\left.\gamma_{k}\right) \sigma^{2} I$, and $\gamma_{k}$ are i.i.d. Bernoulli random variable with $P\left(\gamma_{k}=1\right)=\bar{\gamma}$. Let us define the following information set:

$$
\mathcal{I}_{k} \triangleq\left\{\mathbf{y}^{k}, \gamma^{k}\right\}
$$

where $\mathbf{y}^{k}=\left(y_{k}, y_{k-1}, \ldots, y_{1}\right), \boldsymbol{\gamma}^{k}=\left(\gamma_{k}, \gamma_{k-1}, \ldots, \gamma_{1}\right)$.

Consider also the following cost function:

$J_{N}\left(\mathbf{u}^{N-1}\right)=\mathbb{E}\left[x_{N}^{\prime} W_{N} x_{N}+\sum_{k=0}^{N-1}\left(x_{k}^{\prime} W_{k} x_{k}+u_{k}^{\prime} U_{k} u_{k}\right) \mid \mathcal{I}_{N}\right]$

We now look for control input sequence $\mathbf{u}^{* N-1}$ that minimizes the above functional given that the information $\mathcal{I}_{k}$ is available at time $k$, i.e.

$$
J_{N}^{*}=\min _{\mathbf{u}^{N-1}} J_{N}\left(\mathbf{u}^{N-1}\right)=J_{N}\left(\mathbf{u}^{* N-1}\right)
$$

where $u_{k}^{*}=u_{k}^{*}\left(\mathcal{I}_{k}\right)$ and $\mathcal{I}_{k}$ is defined in Equation 3.

\section{MAThematicAl BACKGROUND}

Before proceeding, let us define the following variables:

$$
\begin{aligned}
\hat{x}_{k \mid k} & \triangleq \mathbb{E}\left[x_{k} \mid \mathcal{I}_{k}\right], \\
e_{k \mid k} & \triangleq x_{k}-\hat{x}_{k \mid k}, \\
P_{k \mid k} & \triangleq \mathbb{E}\left[e_{k \mid k} e_{k \mid k}^{\prime} \mid \mathcal{I}_{k}\right] .
\end{aligned}
$$

In the following derivation we will make use of the following facts

Lemma 1. The following facts are true:

(a) $\mathbb{E}\left[\left(x_{k}-\hat{x}_{k}\right) \hat{x}_{k}^{\prime} \mid \mathcal{I}_{k}\right]=\mathbb{E}\left[e_{k \mid k} \hat{x}_{k}^{\prime} \mid \mathcal{I}_{k}\right]=0$

(b) $\mathbb{E}\left[x_{k}^{\prime} S x_{k} \mid \mathcal{I}_{k}\right]=\hat{x}_{k}^{\prime} S \hat{x}_{k}+\operatorname{trace}\left(S P_{k \mid k}\right), \quad \forall S$

(c) $\mathbb{E}\left[\mathbb{E}\left[g\left(x_{k+1}\right) \mid \mathcal{I}_{k+1}\right] \mid \mathcal{I}_{k}\right]=\mathbb{E}\left[g\left(x_{k+1}\right) \mid \mathcal{I}_{k}\right], \quad \forall g(\cdot)$

Proof: (a) It follows directly from the definition. In fact: $\mathbb{E}\left[\left(x_{k}-\hat{x}_{k}\right) \hat{x}_{k}^{\prime} \mid \mathcal{I}_{k}\right]=\mathbb{E}\left[x_{k} \hat{x}_{k}^{\prime}-\hat{x}_{k} \hat{x}_{k}^{\prime} \mid \mathcal{I}_{k}\right]=$ $\mathbb{E}\left[x_{k} \mid \mathcal{I}_{k}\right] \hat{x}_{k}^{\prime}-\hat{x}_{k} \hat{x}_{k}^{\prime}=0$

(b) Using standard algebraic operations and the previous fact we have:

$$
\begin{aligned}
\mathbb{E}\left[x_{k}^{\prime} S x_{k} \mid \mathcal{I}_{k}\right] & =\mathbb{E}\left[\left(x_{k}-\hat{x}_{k}+\hat{x}_{k}\right)^{\prime} S\left(x_{k}-\hat{x}_{k}+\hat{x}_{k}\right) \mid \mathcal{I}_{k}\right] \\
& =\hat{x}_{k}^{\prime} S \hat{x}_{k}+\mathbb{E}\left[\left(x_{k}-\hat{x}_{k}\right)^{\prime} S\left(x_{k}-\hat{x}_{k}\right)\right]+ \\
& +2 \mathbb{E}\left[\hat{x}_{k}^{\prime} S\left(x_{k}-\hat{x}_{k}\right) \mid \mathcal{I}_{k}\right] \\
& =\hat{x}_{k}^{\prime} S \hat{x}_{k}+2 \operatorname{trace}\left\{S \mathbb{E}\left[\left(x_{k}-\hat{x}_{k}\right) \hat{x}_{k}^{\prime} \mid \mathcal{I}_{k}\right]\right\}+ \\
& +\operatorname{trace}\left\{S \mathbb{E}\left[\left(x_{k}-\hat{x}_{k}\right)\left(x_{k}-\hat{x}_{k}\right)^{\prime} \mid \mathcal{I}_{k}\right]\right\} \\
& =\hat{x}_{k}^{\prime} S \hat{x}_{k}+\operatorname{trace}\left\{S P_{k \mid k}\right\}
\end{aligned}
$$

(c) Let $(X, Y, Z)$ be any random vectors, $g(\cdot)$ any function, and $p$ the probability distribution, then

$$
\begin{aligned}
& \mathbb{E}_{Y, Z}[g(X, Y, Z) \mid X]= \\
& =\int_{Z} \int_{Y} g(X, Y, Z) p(Y, Z \mid X) d Y d Z \\
& =\int_{Z} \int_{Y} g(X, Y, Z) p(Y \mid Z, X) p(Z \mid X) d Y d Z \\
& =\int_{Z}\left[\int_{Y} g(X, Y, Z) p(Y \mid Z, X) d Y\right] p(Z \mid X) d Z \\
& =\mathbb{E}_{Z}\left[\mathbb{E}_{Y}[g(X, Y, Z) \mid Z, X] \mid X\right] .
\end{aligned}
$$


where we used the Bayes' Rule. Since by hypothesis $\mathcal{I}_{k} \subseteq$ $\mathcal{I}_{k+1}$, then fact (c) follows from the above equality by substituting $\mathcal{I}_{k}=X$ and $\mathcal{I}_{k+1}=(X, Z)$.

\section{Finite And Infinite Horizon LQG}

We first start finding the optimal estimator, which will be needed to solve the LQG controller design, as it will be shown later.

\section{A. Estimator Design, $\sigma \rightarrow+\infty$}

We derive the equations for optimal estimator using similar arguments used for the standard Kalman filtering equations. The innovation step is given by:

$$
\begin{aligned}
& \hat{x}_{k+1 \mid k} \triangleq \mathbb{E}\left[x_{k+1} \mid \mathcal{I}_{k}\right]=\mathbb{E}\left[A x_{k}+B u_{k}+w_{k} \mid \mathcal{I}_{k}\right] \\
&=A \mathbb{E}\left[x_{k} \mid \mathcal{I}_{k}\right]+B u_{k} \\
&=A \hat{x}_{k \mid k}+B u_{k} \\
& e_{k+1 \mid k} \triangleq x_{k+1}-\hat{x}_{k+1 \mid k} \\
& P_{k+1 \mid k} \triangleq \mathbb{E}\left[e_{k+1 \mid k} e_{k+1 \mid k}^{\prime} \mid \mathcal{I}_{k}\right]= \\
&=\mathbb{E}\left[\left(A\left(x_{k}-\hat{x}_{k \mid k}\right)+w_{k}\right)\left(A\left(x_{k}-\hat{x}_{k \mid k}\right)+w_{k}\right)^{\prime} \mid \mathcal{I}_{k}\right] \\
&=A \mathbb{E}\left[e_{k \mid k} e_{k \mid k}^{\prime} \mid \mathcal{I}_{k}\right] A^{\prime}+\mathbb{E}\left[w_{k} w_{k}^{\prime}\right] \\
&=A P_{k \mid k} A^{\prime}+Q
\end{aligned}
$$

where we used the independence of $w_{k}$ and $\mathcal{I}_{k}$. Since $y_{k+1}, \gamma_{k+1}, w_{k}$ and $\mathcal{I}_{k}$ are all independent of each other and following the same approach described in [1], then correction step is given by:

$$
\begin{aligned}
\hat{x}_{k+1 \mid k+1} & =\hat{x}_{k+1 \mid k}+\gamma_{k+1} K_{k+1}\left(y_{k+1}-C \hat{x}_{k+1 \mid k}\right)(10) \\
P_{k+1 \mid k+1} & =P_{k+1 \mid k}-\gamma_{k+1} K_{k+1} C P_{k+1 \mid k} \\
K_{k+1} & \triangleq P_{k+1 \mid k} C^{\prime}\left(C P_{k+1 \mid k} C^{\prime}+R\right)^{-1}
\end{aligned}
$$

where we took the limit $\sigma \rightarrow+\infty$.

The initial conditions for the estimator iterative equations are:

$$
\begin{aligned}
& \hat{x}_{0 \mid-1}=0 \\
& P_{0 \mid-1}=P_{0}
\end{aligned}
$$

\section{B. Estimator properties}

Here we compute some quantities that will prove useful when deriving the equation for the optimal LQG controller. Consider first the following variable:

$$
\begin{aligned}
& \Delta_{k+1 \mid k} \triangleq \mathbb{E}\left[\hat{x}_{k+1 \mid k} \hat{x}_{k+1 \mid k}^{\prime} \mid \mathcal{I}_{k}\right]= \\
& =\mathbb{E}\left[\left(A \hat{x}_{k \mid k}+B u_{k}\right)\left(A \hat{x}_{k \mid k}+B u_{k}\right)^{\prime} \mid \mathcal{I}_{k}\right] \\
& =\mathbb{E}\left[A \hat{x}_{k \mid k} \hat{x}_{k \mid k}^{\prime} A^{\prime}+2 A \hat{x}_{k \mid k} u_{k}^{\prime} B^{\prime}+B u_{k} u_{k}^{\prime} B^{\prime} \mid \mathcal{I}_{k}\right] \\
& =A \hat{x}_{k \mid k} \hat{x}_{k \mid k}^{\prime} A^{\prime}+2 A \hat{x}_{k \mid k} u_{k}^{\prime} B^{\prime}+B u_{k} u_{k}^{\prime} B^{\prime}
\end{aligned}
$$

where we used the fact that $\hat{x}_{k \mid k}$ is independent of $\mathcal{I}_{k}$.

Equation (10) can be rewritten as follows:

$$
\begin{aligned}
& \hat{x}_{k+1 \mid k+1}= \\
& =\hat{x}_{k+1 \mid k}+\gamma_{k+1} K_{k+1}\left(C\left(x_{k+1}-\hat{x}_{k+1 \mid k}\right)+v_{k+1}\right) \\
& =\hat{x}_{k+1 \mid k}+\gamma_{k+1} K_{k+1}\left(C e_{k+1 \mid k}+v_{k+1}\right)
\end{aligned}
$$

The first and the second terms in the previous equation and $v_{k+1}$ are independent of each other by Lemma 1 (a) and have zero mean. Moreover, the random variable $\gamma_{k+1}$ is independent of the previous terms. Therefore, we have:

$$
\begin{aligned}
& \Delta_{k+1 \mid k+1} \triangleq \mathbb{E}\left[\hat{x}_{k+1 \mid k+1} \hat{x}_{k+1 \mid k+1}^{\prime} \mid \mathcal{I}_{k}\right]= \\
& =\mathbb{E}\left[\hat{x}_{k+1 \mid k} \hat{x}_{k+1 \mid k}^{\prime} \mid \mathcal{I}_{k}\right]+ \\
& \quad+\mathbb{E}\left[\gamma _ { k + 1 } ^ { 2 } K _ { k + 1 } \left(C e_{k+1 \mid k} e_{k+1 \mid k}^{\prime} C^{\prime}+\right.\right. \\
& \left.\left.\quad+v_{k+1} v_{k+1}\right) K_{k+1}^{\prime} \mid \mathcal{I}_{k}\right]= \\
& =\Delta_{k+1 \mid k}+\mathbb{E}\left[\gamma _ { k + 1 } ^ { 2 } K _ { k + 1 } \left(C e_{k+1 \mid k} e_{k+1 \mid k}^{\prime} C^{\prime}+\right.\right. \\
& \left.\left.\quad+v_{k+1} v_{k+1}\right) K_{k+1}^{\prime} \mid \mathcal{I}_{k}\right]= \\
& =\Delta_{k+1 \mid k}+ \\
& \quad+\mathbb{E}\left[\gamma_{k+1}^{2} K_{k+1}\left(C P_{k+1 \mid k} C^{\prime}+R\right) K_{k+1}^{\prime} \mid \mathcal{I}_{k}\right]= \\
& =\Delta_{k+1 \mid k}+ \\
& \quad+\mathbb{E}\left[\gamma_{k+1}^{2} P_{k+1 \mid k} C^{\prime}\left(C P_{k+1 \mid k} C^{\prime}+R\right)^{-1} C P_{k+1 \mid k}^{\prime} \mid \mathcal{I}_{k}\right]= \\
& =\Delta_{k+1 \mid k}+\mathbb{E}\left[M_{k+1} \mid \mathcal{I}_{k}\right] \\
& M_{k+1} \triangleq \gamma_{k+1}^{2} P_{k+1 \mid k} C^{\prime}\left(C P_{k+1 \mid k} C^{\prime}+R\right)^{-1} C P_{k+1 \mid k}^{\prime}
\end{aligned}
$$

where $M_{k+1}$ is a stochastic matrix which depends on the sequence $\left\{\gamma_{k}\right\}$, but is independent of $u_{k}$.

\section{Controller design}

To derive the optimal feedback control law and the corresponding value for the objective function we will follow the dynamic programming approach based on the cost-to-go iterative procedure.

Define the optimal value function $V_{k}\left(x_{k}\right)$ as follows:

$$
\begin{gathered}
V_{N}\left(x_{N}\right) \triangleq \mathbb{E}\left[x_{N}^{\prime} W_{N} x_{N} \mid \mathcal{I}_{N}\right] \\
V_{k}\left(x_{k}\right) \triangleq \min _{u_{k}} \mathbb{E}\left[x_{k}^{\prime} W_{k} x_{k}+u_{k}^{\prime} U_{k} u_{k}+V_{k+1}\left(x_{k+1}\right) \mid \mathcal{I}_{k}\right]
\end{gathered}
$$

Using dynamic programming theory [16] [17], one can show that $J_{N}^{*}=V_{0}\left(x_{0}\right)$.

We claim that the value function $V_{k}\left(x_{k}\right)$ can be written as:

$$
V_{k}\left(x_{k}\right)=\hat{x}_{k \mid k}^{\prime} S_{k} \hat{x}_{k \mid k}+c_{k}, \quad k=0, \ldots, N
$$

where the matrix $S_{k}$ and the scalar $c_{k}$ are to be determined and are independent of the information set $\mathcal{I}$. We will prove it by induction. The claim is certainly true for $k=N$, in fact:

$$
\begin{aligned}
V_{N}\left(x_{N}\right) & =\mathbb{E}\left[x_{N}^{\prime} W_{N} x_{N} \mid \mathcal{I}_{N}\right] \\
& =\hat{x}_{N \mid N}^{\prime} W_{N} x_{N \mid N}+\operatorname{trace}\left(W_{N} P_{N \mid N}\right)
\end{aligned}
$$

where we used Lemma 1 (b), with the following choice of parameters:

$$
\begin{aligned}
S_{N} & =W_{N} \\
c_{N} & =\operatorname{trace}\left(W_{N} P_{N \mid N}\right)
\end{aligned}
$$

Suppose now that the claim is true for $k+1$, i.e. $V_{k+1}\left(x_{k+1}\right)=\hat{x}_{k+1 \mid k+1}^{\prime} S_{k+1} \hat{x}_{k+1 \mid k+1}+c_{k+1}$, and we use it to compute the value function at time step $k$ as follows: 


$$
\begin{aligned}
& V_{k}\left(x_{k}\right)= \\
& =\min _{u_{k}} \mathbb{E}\left[x_{k}^{\prime} W_{k} x_{k}+u_{k}^{\prime} U_{k} u_{k}+V_{k+1}\left(x_{k+1}\right) \mid \mathcal{I}_{k}\right] \\
& =\min _{u_{k}} \mathbb{E}\left[x_{k}^{\prime} W_{k} x_{k}+u_{k}^{\prime} U_{k} u_{k}+\right. \\
& \left.+\quad \mathbb{E}\left[x_{k+1 \mid k+1}^{\prime} S_{k+1} x_{k+1 \mid k+1}+c_{k+1} \mid \mathcal{I}_{k+1}\right] \mid \mathcal{I}_{k}\right] \\
& =\min _{u_{k}} \mathbb{E}\left[x_{k}^{\prime} W_{k} x_{k}+u_{k}^{\prime} U_{k} u_{k}+\right. \\
& \left.+x_{k+1 \mid k+1}^{\prime} S_{k+1} x_{k+1 \mid k+1}+c_{k+1} \mid \mathcal{I}_{k}\right] \\
& =\min _{u_{k}}\left(\hat{x}_{k \mid k}^{\prime} W_{k} \hat{x}_{k \mid k}+\operatorname{trace}\left(W_{k} P_{k \mid k}\right)+u_{k}^{\prime} U_{k} u_{k}+\right. \\
& \left.+\operatorname{trace}\left(S_{k+1} \mathbb{E}\left[x_{k+1 \mid k+1} x_{k+1 \mid k+1}^{\prime} \mid \mathcal{I}_{k}\right]+\mathbb{E}\left[c_{k+1} \mid \mathcal{I}_{k}\right]\right)\right) \\
& =\min _{u_{k}}\left(u_{k}^{\prime} U_{k} u_{k}+\operatorname{trace}\left(S_{k+1} \Delta_{k+1 \mid k}\right)\right)+ \\
& +\operatorname{trace}\left(S_{k+1} M_{k+1}\right)+\hat{x}_{k \mid k}^{\prime} W_{k} \hat{x}_{k \mid k}+ \\
& +\quad \operatorname{trace}\left(W_{k} P_{k \mid k}\right)+\mathbb{E}\left[c_{k+1} \mid \mathcal{I}_{k}\right] \\
& =\min _{u_{k}}\left(u_{k}^{\prime} U_{k} u_{k}+u_{k}^{\prime} B^{\prime} S_{k+1} B u_{k}+2 u_{k}^{\prime} B^{\prime} S_{k+1} A \hat{x}_{k \mid k}\right)+ \\
& +\hat{x}_{k \mid k}^{\prime} A^{\prime} S_{k+1} A \hat{x}_{k \mid k}+\operatorname{trace}\left(S_{k+1} M_{k+1}\right)+ \\
& +\hat{x}_{k \mid k}^{\prime} W_{k} \hat{x}_{k \mid k}+\operatorname{trace}\left(W_{k} P_{k \mid k}\right)+\mathbb{E}\left[c_{k+1} \mid \mathcal{I}_{k}\right]
\end{aligned}
$$

where we used Lemma 1(c) in the third line and Lemma 1(b) in the fourth line. We substituted Equations (17) in fifth line and we separated terms which did not depend on $u_{k}$. Finally, in sixth line we substituted Equations (15) and we used the property $\operatorname{trace}(A B)=\operatorname{trace}(B A)$. The value function is a convex quadratic function of the input, therefore the minimizer can be simply obtained by solving $\frac{\partial V_{k}}{\partial u_{k}}=0$, which gives:

$$
u_{k}=-\left(B^{\prime} S_{k+1} B+U_{k}\right)^{-1} B^{\prime} S_{k+1} A \hat{x}_{k \mid k}=L_{k} \hat{x}_{k \mid k} .
$$

The optimal feedback is therefore a simple linear feedback of the estimated state. If we substitute the minimizer back into Equation (23) and we use Equation (19) we get:

$$
\begin{aligned}
& V_{k}\left(x_{k}\right)= \\
& =-\hat{x}_{k \mid k}^{\prime} A^{\prime} S_{k+1} B\left(B^{\prime} S_{k+1} B+U_{k}\right)^{-1} B^{\prime} S_{k+1} A \hat{x}_{k \mid k}+ \\
& \quad+\hat{x}_{k \mid k}^{\prime} A^{\prime} S_{k+1} A \hat{x}_{k \mid k}+\hat{x}_{k \mid k}^{\prime} W_{k} \hat{x}_{k \mid k}+ \\
& \quad+\operatorname{trace}\left(S_{k+1} M_{k}\right)+\operatorname{trace}\left(W_{k} P_{k \mid k}\right)+\mathbb{E}\left[c_{k+1} \mid \mathcal{I}_{k}\right],
\end{aligned}
$$

and

$$
\begin{aligned}
& \hat{x}_{k \mid k}^{\prime} S_{k} \hat{x}_{k \mid k}+c_{k}=\hat{x}_{k \mid k}^{\prime}\left(A^{\prime} S_{k+1} A+W_{k}-\right. \\
& \left.\quad+A^{\prime} S_{k+1} B\left(B^{\prime} S_{k+1} B+U_{k}\right)^{-1} B^{\prime} S_{k+1} A\right) \hat{x}_{k \mid k}+ \\
& \quad+\operatorname{trace}\left(S_{k+1} M_{k+1}\right)+\operatorname{trace}\left(W_{k} P_{k \mid k}\right)+\mathbb{E}\left[c_{k+1} \mid \mathcal{I}_{k}\right]
\end{aligned}
$$

For the previous equation to hold for $\hat{x}_{k \mid k}$, we need to have:

$$
\begin{aligned}
S_{k} & =A^{\prime} S_{k+1} A+W_{k}- \\
& +A^{\prime} S_{k+1} B\left(B^{\prime} S_{k+1} B+U_{k}\right)^{-1} B^{\prime} S_{k+1} A \\
c_{k} & =\mathbb{E}\left[c_{k+1} \mid \mathcal{I}_{k}\right]+\operatorname{trace}\left(S_{k+1} M_{k+1}\right)+\operatorname{trace}\left(W_{k} P_{k \mid k}\right)
\end{aligned}
$$
by:

Therefore, the cost function for the optimal LQG is given

$$
\begin{aligned}
& J_{N}^{*}=V_{0}\left(x_{0}\right)=\hat{x}_{0 \mid 0}^{\prime} S_{0} \hat{x}_{0 \mid 0}+ \\
& +\sum_{k=0}^{N}\left(\operatorname{trace}\left(S_{k+1} \mathbb{E}_{\gamma}\left[M_{k+1}\right]\right)+\operatorname{trace}\left(W_{k} \mathbb{E}_{\gamma}\left[P_{k \mid k}\right]\right)(26)\right.
\end{aligned}
$$

where the first term is zero since by hypothesis $\hat{x}_{0}=0$. The matrices $M_{k+1}$ and $P_{k \mid k}$ in the second and third term are stochastic since they are function of the sequence $\left\{\gamma_{k}\right\}$, where the expectation with respect to this process is made explicit in $\mathbb{E}_{\gamma}$. The exact expected value of these matrices cannot be computed analytically, as shown in [1], but it can be bounded by computable quantities. In fact let us consider the following three equations:

$$
\begin{aligned}
\widehat{P}_{k+1 \mid k} & =A \widehat{P}_{k \mid k-1} A^{\prime}+Q- \\
& +\bar{\gamma} A \widehat{P}_{k \mid k-1} C^{\prime}\left(C \widehat{P}_{k \mid k-1} C^{\prime}+R\right)^{-1} C \widehat{P}_{k \mid k-1} A^{\prime} \\
\widehat{P}_{k+1 \mid k+1} & =\widehat{P}_{k \mid k-1}- \\
& +\bar{\gamma} \widehat{P}_{k \mid k-1} C^{\prime}\left(C \widehat{P}_{k \mid k-1} C^{\prime}+R\right)^{-1} C \widehat{P}_{k \mid k-1} \\
\widetilde{P}_{k+1 \mid k} & =(1-\bar{\gamma}) A \widetilde{P}_{k \mid k-1} A^{\prime}+Q
\end{aligned}
$$

initialized to $\widehat{P}_{0 \mid-1}=\widetilde{P}_{0 \mid-1}=P_{0}$. It is possible to show that:

$$
\begin{aligned}
\widetilde{P}_{k \mid k} & \leq \mathbb{E}_{\gamma}\left[P_{k \mid k}\right] \leq \widehat{P}_{k \mid k} \\
0 & \leq \mathbb{E}_{\gamma}\left[M_{k+1}\right] \leq \\
& \leq \widehat{P}_{k \mid k-1} C^{\prime}\left(C \widehat{P}_{k \mid k-1} C^{\prime}+R\right)^{-1} C \widehat{P}_{k \mid k-1} \\
& =\widehat{P}_{k \mid k-1}-\widehat{P}_{k+1 \mid k+1}
\end{aligned}
$$

Therefore we have:

$$
\begin{aligned}
J_{N}^{\min } & \leq J_{N}^{*} \leq J_{N}^{\max } \\
J_{N}^{\max } & =\sum_{k=0}^{N}\left(\operatorname{trace}\left(S_{k+1}\left(\widehat{P}_{k \mid k-1}-\widehat{P}_{k+1 \mid k+1}\right)\right)+\right. \\
& \left.+\operatorname{trace}\left(W_{k} \widehat{P}_{k \mid k}\right)\right) \\
J_{N}^{\min } & =\sum_{k=0}^{N} \operatorname{trace}\left(W_{k} \widetilde{P}_{k \mid k}\right)
\end{aligned}
$$

\section{Finite and Infinite Horizon LQG control}

The previous equations were derived for the finite horizon LQG. The infinite horizon LQG can be obtained by taking the limit for $N \rightarrow+\infty$ of the previous equations. However, the matrices $\left\{M_{k+1}\right\}$ and $\left\{P_{k \mid k}\right\}$ depend on the specific realization of the observation sequence $\left\{\gamma_{k}\right\}$, therefore the minimal cost $J_{N}$ is a stochastic function and does not have a limit. Differently from standard LQG controller design where the controller always stabilizes the original system, in the case of control with packet losses, the stability can be lost if the arrival probability $\bar{\gamma}$ is below a certain threshold. In particular the Equation for the cost matrix $S_{k}$ is the solution of a modified Riccati Algebraic Equation (MARE) which was already introduced and studied in our previous work [1]. In particular, Equation (25) is the dual of the estimator equation presented in [1]. Therefore, the same conclusions can be drawn and we are now ready summarize the previous result in the following theorem:

Theorem 1 (Finite Horizon LQG). Consider the system (1)-(2) and consider the problem of minimizing the cost function (4) with policy $u_{k}=f\left(\mathcal{I}_{k}\right)$, where $\mathcal{I}_{k}$ is the information available under the communication model, as defined in Equation (3). Then, the optimal control is a linear 
function of the estimated system state given by Equation (24), where the matrix $S_{k}$ can be computed iteratively using Equation (25). The separation principle still holds under our assumptions, since the optimal estimator is independent of the control input $u_{k}$. The optimal state estimator is given by Equations (7)-(10) and (9)-(12), and the minimal achievable cost is given given by Equation (26).

Theorem 2 (Infinite Horizon LQG). Consider the same systems as defined in the previous theorem with the following additional hypothesis: $W_{N}=W_{k}=W$ and $U_{k}=U$. Moreover, let $(A, B)$ and $\left(A, Q^{\frac{1}{2}}\right)$ be controllable, and let $(A, C)$ and $\left(A, W^{\frac{1}{2}}\right)$ be observable. Let us consider the limiting case $N \rightarrow+\infty$, then, there exists a critical arrival probability $\gamma_{\min }$ which satisfies the following property:

$$
\min \left(1,1-\frac{1}{\left|\lambda_{\max }(A)\right|^{2}}\right) \leq \gamma_{\min } \leq 1
$$

where $\left|\lambda_{\max }(A)\right|$ is the eigenvalue of matrix $A$ with largest absolute value, such that for all $\bar{\gamma}>\gamma_{\min }$ we have:

$$
\begin{aligned}
& L_{k}=L_{\infty}=-\left(B^{\prime} S_{\infty} B+U\right)^{-1} B^{\prime} S_{\infty} A \\
& \frac{1}{N} J_{N}^{\min } \leq \frac{1}{N} J_{N}^{*} \leq \frac{1}{N} J_{N}^{\max }
\end{aligned}
$$

where the matrix $S_{\infty}$, and the mean cost bounds $J_{N}^{\min }, J_{N}^{\max }$ are given by:

$$
\begin{aligned}
& \lim _{N \rightarrow+\infty} \frac{1}{N} J_{N}^{\max }=J_{\infty}^{\max }= \\
& =\bar{\gamma} \operatorname{trace}\left(\left(S_{\infty}-W\right) \bar{P}_{\infty} C^{\prime}\left(C \bar{P}_{\infty} C^{\prime}+R\right)^{-1} C \bar{P}_{\infty}+\right. \\
& \left.+\operatorname{trace}\left(W \bar{P}_{\infty}\right)\right) \\
& \lim _{N \rightarrow+\infty} \frac{1}{N} J_{N}^{\text {min }}=J_{\infty}^{\text {min }}=\operatorname{trace}\left(W \underline{P}_{\infty}\right),
\end{aligned}
$$

and the matrices $S_{\infty}, \bar{P}_{\infty}, \underline{P}_{\infty}$ are given by:

$$
\begin{aligned}
S_{\infty} & =A^{\prime} S_{\infty} A+W- \\
& +A^{\prime} S_{\infty} B\left(B^{\prime} S_{\infty} B+U\right)^{-1} B^{\prime} S_{\infty} A \\
\bar{P}_{\infty} & =A \bar{P}_{\infty} A^{\prime}+Q- \\
& +\bar{\gamma} A \bar{P}_{\infty} C^{\prime}\left(C \bar{P}_{\infty} C^{\prime}+R\right)^{-1} C \bar{P}_{\infty} A^{\prime} \\
\underline{P}_{\infty} & =(1-\bar{\gamma}) A \underline{P}_{\infty} A^{\prime}+Q,
\end{aligned}
$$

where $S_{\infty}$ is the solution of a standard ARE which always exists [18], $\bar{P}_{\infty}$ is the solution of a MARE dual to the one proposed in [1], and $\underline{P}_{\infty}$ is the solution of a Lyapunov Equation. Moreover, the assumptions above are necessary and sufficient conditions for the closed loop system under $L Q G$ feedback to be stable in mean sense. The critical probability $\gamma_{\min }$ can be computed via the solution of the following LMIs optimization problem:

$$
\begin{aligned}
& \gamma_{\text {min }}=\operatorname{argmin}_{\bar{\gamma}} \Psi_{\gamma}(Y, Z)>0, \quad 0 \leq Y \leq I . \\
& \Psi_{\gamma}(Y, Z)= \\
& =\left[\begin{array}{ccc}
Y & \sqrt{\gamma}(Y A+Z C) & \sqrt{1-\gamma} Y A \\
\sqrt{\gamma}\left(A^{\prime} Y+C^{\prime} Z^{\prime}\right) & Y & 0 \\
\sqrt{1-\gamma} A^{\prime} Y & 0 & Y
\end{array}\right]
\end{aligned}
$$

\section{CONCLUSION}

Motivated by applications where control is performed over a communication network, in this paper we extend our previous results on estimation with intermittent observations to the optimal control problem. First, we show that the separation principle holds also in the case when the observed state can be lost at each time step with some probability $\lambda$. Then, we show how the optimal control problem formally reduces to the solution of a standard Riccati equation for the controller and the same modified Riccati equation that was studied in [1], [8] for the estimator. Accordingly, we provide upper and lower bounds on the expected optimal cost functional and characterize its convergence conditions, showing a transition to an unbounded cost beyond a critical arrival probability. We also provide upper and lower bounds for the cost in the finite horizon case. Future work will include stability analysis and LQG controller design for a more general class of systems, specifically the ones where also loss of control packets occurs between the controller and one or more actuators.

\section{REFERENCES}

[1] B. Sinopoli, L. Schenato, M. Franceschetti, K. Poolla, M. Jordan, and S. Sastry, "Kalman filtering with intermittent observations," IEEE Transactions on Automatic Control, September 2004, to appear.

[2] Smart dust project home page. http://robotics.eecs.berkeley.edu/ pister/SmartDust/.

[3] NEST project at Berkeley home page. http://webs.cs.berkeley.edu/nest-index.html.

[4] Seismic sensor research at berkeley, home page. http://www.berkeley.edu/news/media/releases /2001/12/13_snsor.html.

[5] P. Varaiya, "Smart cars on smart roads: Problems of control," IEEE Transactions on Automatic Control, vol. 38(2), February 1993.

[6] J. Lygeros, D. N. Godbole, and S. S. Sastry, "Verified hybrid controllers for automated vehicles," IEEE Transactions on Automatic Control, vol. 43(4), 1998.

[7] B. Sinopoli, C. Sharp, S. Schaffert, L. Schenato, and S. Sastry, "Distributed control applications within sensor networks," IEEE Proceedings Special Issue on Distributed Sensor Networks, November 2003.

[8] B. Sinopoli, L. Schenato, M. Franceschetti, K. Poolla, M. Jordan, and S. Sastry, "Kalman filtering with intermittent observations," in IEEE Conference on Decision and Control, December 2003.

[9] J. Nilsson, "Real-time control systems with delays," Ph.D. dissertation, Department of Automatic Control, Lund Institute of Technology, 1998.

[10] C. N. Hadjicostis and R. Touri, "Feedback control utilizing packet dropping network links," in Proceedings of the 41st IEEE Conference on Decision and Control, Las Vegas, NV, Dec 2002, invited.

[11] Q. Ling and M. Lemmon, "Soft real-time scheduling of networked control systems with dropouts governed by a markov chain," in American Control Conference, June 2003, denver, CO.

[12] - "Optimal dropout compensation in networked control systems," in IEEE conference on decision and control, December 2003, maui, HI.

[13] M. Mariton, Jump Linear Systems in Automatic Control. Marcel Dekker, 1990.

[14] M. Athans, R. Ku, and S. B. Gershwin, "The uncertainty threshold principle, some fundamental limitations of optimal decision making under dynamic uncertainty," IEEE Transactions on Automatic Control, vol. 22(3), pp. 491-495, June 1977.

[15] R. Ku and M. Athans, "Further results on the uncertainty threshold principle," IEEE Transactions on Automatic Control, vol. 22(5), pp. 491-495, October 1977. 
[16] P. Kumar and P. Varaiya, Stochastic Systems: Estimation, Identification and Adaptive Control, ser. Information and System Science Series, T. Kailath, Ed. Englewood Cliffs, NJ 07632: Prentice Hall, 1986.

[17] D. Bertsekas and J. Tsitsiklis, Neuro-Dynamic Programming. Athena Scientific, September 1996.

[18] P. S. Maybeck, Stochastic models, estimation, and control, ser. Mathematics in Science and Engineering, 1979, vol. 141. 